\title{
THE CORRELATION BETWEEN JOB-HOPPING ATTITUDE AND TURNOVER BEHAVIOR: A JOB SATISFACTION PERSPECTIVE IN BANGLADESH
}

\author{
Md. Chapol Ali \\ PhD Fellow \\ Department of Business Administration \\ Wonkwang University, South Korea \\ E-mail: chapol1344@gmail.com \\ K. M. Anwarul Islam \\ Associate Professor \\ Department of Business Administration \\ The Millennium University, Dhaka, Bangladesh \\ E-mail: ai419bankingdu@gmail.com \\ Soo-Jin Chung \\ Professor \\ Department of Business Administration \\ Wonkwang University, South Korea \\ E-mail: sj@wku.ac.kr \\ Nurul Mohammad Zayed \\ Assistant Professor \& Head \\ Department of Real Estate \\ Daffodil International University, Dhaka, Bangladesh \\ E-mail: zayed.bba@daffodilvarsity.edu.bd \\ Mohammad Rakibul Islam \\ PhD Fellow \\ Department of Business Administration \\ Wonkwang University, South Korea \\ E-mail: rakibul.islam0@gmail.com
}

\begin{abstract}
A worker is a company's principal power base. Recently, the employment and turnover rates of employees have risen very high for several reasons. It was still a matter of great importance to organizations. This study focuses on the multiple reasons for work saving and turnover behaviors at different workers levels in Bangladesh. This research focuses on various factors. Through this hypothesis, the key methods to determine the independent variables are studied, including worker satisfaction, job quality, employee facilities, and employee dependent
\end{abstract}


variables, employment and turnover rates. A correlation between the independent and dependent variables has been identified. A survey of 150 respondents working at various levels in Bangladeshi organizations was performed with the hypothesis-based questioner. The data obtained through the survey and statistically analyzed using tools for data analysis (SPSS). In this study, the relationship between independent variables and dependent variables was analyzed through descriptive statistics, reliability analysis and inference analysis. The study of linear and multi-linear regression on these parameters indicates the purpose of the reversal. Based on the results; the theoretical issues will be validated. This study explores the key explanation for this and establishes a research paradigm to minimize Bangladesh's high turnover.

Keyword: Bangladesh, Worker Satisfaction, Job Quality, Employee Facilities, Multi-Linear Regression.

JEL Classification Codes: J62, J28, J63.

\section{INTRODUCTION}

This job-hopping and turnover intent is a widespread phenomenon in the modern era. According to Huak, Pivi, and Hassan (2015), the trend of transitioning from one profession to another is driven by an intermediate desire, which urges employees to make these changes without considering the rationale. Moreover, the purpose of turnover has always been the main focus of today's companies. It has become an important and challenging issue for all types of businesses due to its negative impact on management (Belete, 2018; Chan, Yeoh, \& Osman, 2010). Turnover of state employees is also a severe problem, especially in human resource management. Employers need to provide economical methods that can satisfy basic needs and have an excellent working atmosphere, particularly for entry-level workers, because the skills of specific individuals aren't all that distinctive. As a rule, a businessman/woman would have two: The ability to improve productivity and decrease the rate of workforce turnover. Both are lucrative endeavors. Direct costs are used for recruiting, job placement, and orientation and preparation. Indirect expenses, the effect on new jobs, the strain on the general labor market and social resources, and further staff turnover will mean its targets. As a result, it is important to investigate staff attrition to assist the company with problem-solving and monitoring, gather the facts, and devise potential solutions.

\section{LITERATURE REVIEW}

Both problems surrounding workforce turnover have been extensively investigated; however, it is yet to be seen what effect it has because of its relation to results, which is a significant concern to business leaders. The work in short-wide swings in the direction of jobs in an organization, regardless of how big or small (Lee \& Mitchell, 2001). Most Bangladesh organizations have difficulty keeping staff members because they cannot discern the reasons for worker satisfaction and commitment (Leedy \& Ormrod, 2001). The employee turn-over crisis is due to some factors. The labor market has changed over the last few decades, and these days it's very typical for anyone between the ages of twenty and thirty to switch jobs regularly. Employment flexibility is more related to "Generation Y" (the Millennials (Generation Y). More than half of the population consists of those in Generation Y (age 25-39) in Malaysia (Cranny, Smith \& Stone, 2016). 


\section{Job Satisfaction and Turnover Intention}

The organizational behavior and management literature is replete with studies on job satisfaction. When workers are involved in work satisfaction, it goes hand in hand with participation, dedication, absenteeism, competitiveness, and organizational commitment. People are vital to any company. Because of this, there has been an increased amount of attention on workers, especially over the past few decades (Krishnan, 2012). On top of that, thanks to these organizations' greater emphasis on human resources, all the problems relating to them have begun focusing on employee issues. Work values are the most critical condition for an organization to understand what a person does at work. Very few organizations have employees who start their careers and finish them as numbers. Most citizens have switched their occupations and workplaces around because of various factors, but the main reasons are varied (Kirschenbaum \& Rita, 1999). This study aims to determine the numerous reasons for staff satisfaction and attrition in the Bangladeshi public and private sectors. The main purpose of this study is to discover the key causes of employee attrition and identify which causes the most important. To Show the correlation between previously reported studies and the variables that predict employee turnover (Lawrence, 2003).

\section{METHOD}

Based on this analysis, it's been found that the decision points to the inference that it is a progressive one. If a person leaves a job because of dissatisfaction and situations, these expectations have to be considered. Employees go through a process to decide to resign, while intentions factor into determining whether or not to leave and begin on a whim and gain strength with time. People try to go to make more time for their other desires. They give up on breaking from their duties. Many researchers claim that employees' willingness to leave usually arises when they perceive their working conditions to be disadvantageous. It was a book that took nine months to write, nine years to design, and one month to implement (Arshad, 2013).
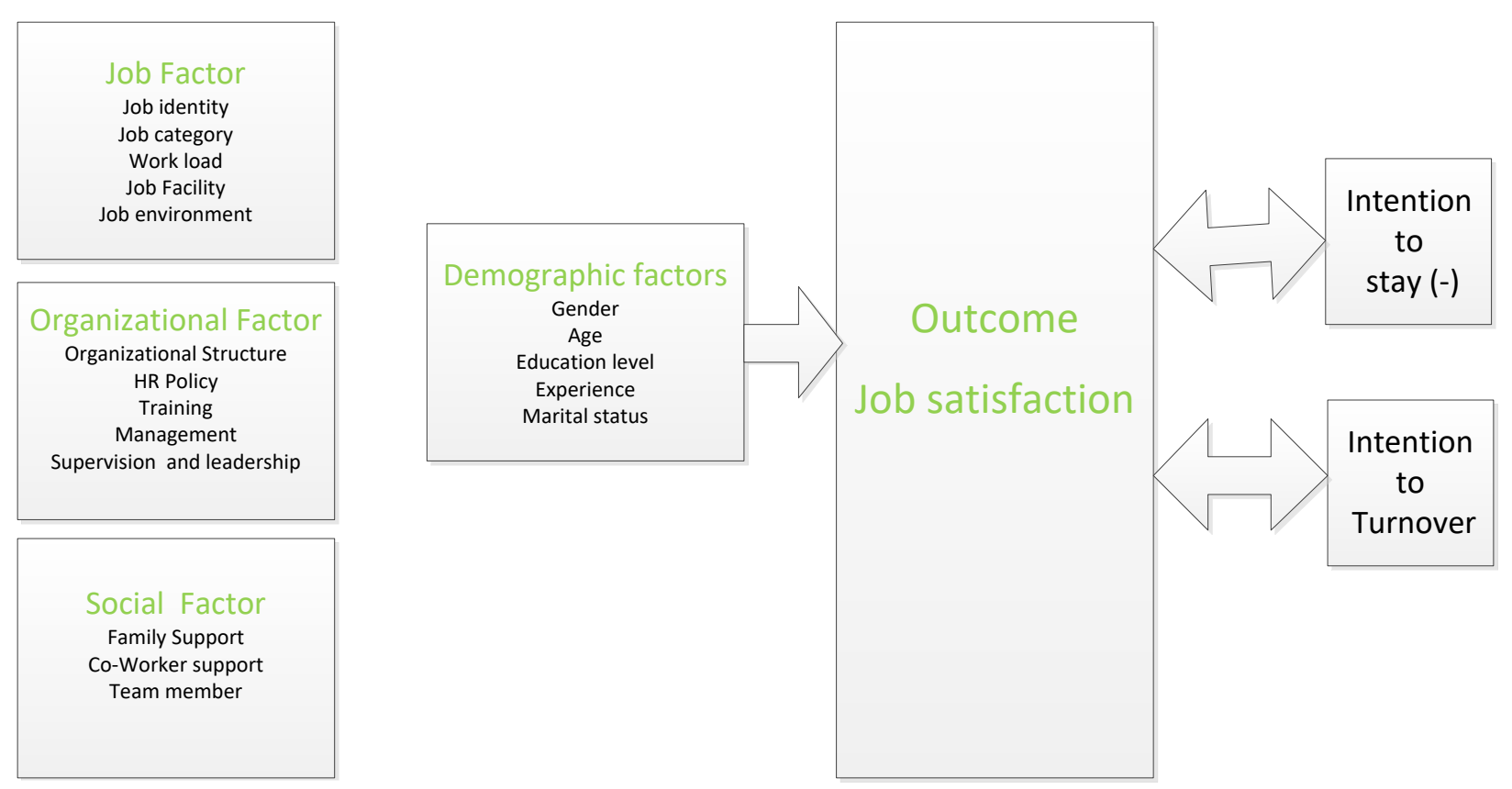

Figure 1. The hypothesized relationship between job satisfaction and turnover intention (Source: Authors' Compilation) 


\section{Analysis Method and Sample Characteristic}

The statistical analysis software (SPSS) used in this study has verified the reliability and validity of the variables to ensure the feasibility of the research results. Correlation analysis is used to validate the functionality between variables. This study adopts the questionnaire with Bangladeshi employees of different organizations. The employees work in the private and public sectors. The survey was conducted mainly by distributing questionnaires on the internet to collect required data conveniently in a short time. The survey time was fromMarch 1, 2020, to June15, 2020. A total of 200 participants submitted their survey after deleting observations with missing data. The final sample contained 149 employees, which can be used as the data for the study. The sample was $24.7 \%$ female and $75.3 \%$, male. $83.3 \%$ of respondents work in the private sector and $16.7 \%$ work in the public sector. Of the sample, $46.9 \%$ of people are over 25 years old, 70.7.7\% were married. The responder answers the survey based on the present job condition and job satisfaction. The sample distribution characteristics are obtained by analyzing the sample data (Table 1).

Table 1. The Sample Characteristic

\begin{tabular}{|c|c|c|c|c|c|}
\hline \multicolumn{2}{|c|}{ Category } & Proportion & \multicolumn{2}{|c|}{ Category } & \multirow{2}{*}{\begin{tabular}{|l|} 
Proportion \\
(27) $16.7 \%$ \\
\end{tabular}} \\
\hline \multirow[t]{2}{*}{ Gender } & Male & (113) $75.3 \%$ & \multirow{2}{*}{$\begin{array}{l}\text { Organization } \\
\text { Type }\end{array}$} & Public & \\
\hline & Female & (37) $24.7 \%$ & & Private & (125) $83.3 \%$ \\
\hline \multirow{7}{*}{ Age } & $\begin{array}{l}25 \text { years old } \\
\text { and below }\end{array}$ & (14)09.39\% & \multirow{5}{*}{ Position } & $\begin{array}{l}\text { junior } \\
\text { lecturer }\end{array}$ & (01) $0.7 \%$ \\
\hline & $\begin{array}{l}\text { 26-30 Years } \\
\text { old }\end{array}$ & (70) $46.98 \%$ & & Lecturer & (01) $0.7 \%$ \\
\hline & $\begin{array}{l}31-35 \text { Years } \\
\text { Old }\end{array}$ & $(34) 22.81 \%$ & & $\begin{array}{l}\text { Non- } \\
\text { Executive } \\
\text { level }\end{array}$ & (23) $15.3 \%$ \\
\hline & $\begin{array}{l}36-40 \text { Years } \\
\text { Old }\end{array}$ & $(15) 10.06 \%$ & & $\begin{array}{l}\text { Executive } \\
\text { level }\end{array}$ & (144) $76 \%$ \\
\hline & $\begin{array}{l}41-45 \text { Years } \\
\text { Old }\end{array}$ & (6) $4.02 \%$ & & $\begin{array}{l}\text { Managerial } \\
\text { level }\end{array}$ & (11) $7.3 \%$ \\
\hline & $\begin{array}{l}46-50 \text { years } \\
\text { old }\end{array}$ & (1) $0.67 \%$ & \multirow{6}{*}{$\begin{array}{l}\text { Work } \\
\text { Number of } \\
\text { Companies }\end{array}$} & 0-1 Years & (31) $20.7 \%$ \\
\hline & $\begin{array}{l}50 \text { Years } \\
\text { above }\end{array}$ & (3) $2.13 \%$ & & 1-2 Years & (42) $28 \%$ \\
\hline \multirow[t]{2}{*}{ Marital status } & Single & (44) $29.3 \%$ & & 2-3 Years & (27) $18 \%$ \\
\hline & Married & (106) $70.7 \%$ & & 3-4 Years & (18) $12 \%$ \\
\hline \multirow{4}{*}{$\begin{array}{l}\text { Educational } \\
\text { Level }\end{array}$} & Diploma & (4) $2.7 \%$ & & 4-5 Years & (7) $4.7 \%$ \\
\hline & Bachelor & (89) $59.3 \%$ & & $\begin{array}{l}5 \text { Years } \\
\text { above }\end{array}$ & (5) $3.4 \%$ \\
\hline & $\begin{array}{l}\text { Master and } \\
\text { above }\end{array}$ & (48) $32 \%$ & & & \\
\hline & $\begin{array}{l}\text { Professional } \\
\text { qualification }\end{array}$ & (9) $6 \%$ & & & \\
\hline
\end{tabular}

Source: Estimated 


\section{Hypotheses}

Hypothesis 1 . The significant compression between public and private sector job satisfaction and employee turnover in Bangladesh.

Hypothesis 2. The demographic factors that are related to job satisfaction and turnover intention. Yes, or Not?

Hypothesis 3. Better job opportunity opportunities, HR Policies, and Practices are positively related to job hopper's intentions to exit an organization.

Hypothesis 4. Pay and benefits motivate job hoppers to select a job or stay in the current organization.

Hypothesis 5. Job factor, Organizational and Social aspect are directly involved in Job satisfaction and Turnover intention

\section{Hypothesis - 1}

The significant relationship between public and private sector job satisfaction and employee turnover in Bangladesh

This is the first hypothesis that contrasts Bangladeshi workers' work satisfaction and business intentions. Data collection by the staff of numerous private companies and public organizations was used in questionnaires. The findings indicate that public employees are different from remote employees because they work with satisfaction and sales intentions. First, Bangladeshi state employees are weaker than private-sector peers regarding international work satisfaction and low attrition intentions.

Secondly, there is a weaker link in public than in private workers between external satisfaction and turnover intention. To increase morale, Bangladeshi public sector administrators should boost the external work satisfaction of their workers and aid unsatisfied people transfers to more happy jobs. They will also want to look at whether their attrition rates are too poor to promote healthier mobility of workers in their organizations.

\section{Hypothesis - 2}

The demographic factors that are related to Job satisfaction and turnover intention. Yes, or Not?

The second hypothesis analysis the relationship between demographic factors like Age, Gender, etc., to job satisfaction and employee turnover. The results show no factor related to the demographic factors for employee turnover and job satisfaction.

\section{Hypothesis - 3}

Better job opportunity opportunities, HR Policies, and Practices are positively related to job hopper's intentions to exit an organization.

Better job opportunities and distribution of HR policy and practice scores are not the same in age and organization scores but the same in gender, experience, qualifications, function, and position 
scores. This suggests that HR policies and practices directly impact employees' willingness to stay and that the effects vary from 23-38-year old's and the organization they are currently involved with. Thus, assumptions are maintained. The nature of the tabulated reactions expresses the strength of the response by supporting the hypothesis.

\section{Hypothesis - 4}

Pay and benefits are a motivating factor for job hoppers for selecting a job or continue to stay in the current organization.

The distribution of pay and benefit scores is equal to age, gender, experience, qualifications, function, and location scores and not similar to company scores. The test results show that employees emphasize pay and benefits, which vary from company to company. If job satisfaction is achieved through progressive HR policy, investment in training and innovation in the workplace, etc., employees balance salary and benefit priorities.

\section{Hypothesis - 5}

Job factors, Organizational and Social factors are directly involved in Job satisfaction and Turnover intention.

This hypothesis shows that the job and social factors are directly involved in Job satisfaction and Turnover intention.

\section{Reliability and Validity Test}

\section{RESULTS \& DISCUSSIONS}

This study used continuous internal coefficients to test reliability, and Cronbach's alpha was above 0.7. Furthermore, Exploration Factor Analysis (EFA) was used to test the validity of this study. All communalism was above 0.5, KMO values were all above 0.8, and Bartlett's sphere of importance level test was within $\mathrm{p}<0.05$, proving that the degree of reliability and effectiveness were all excellent. The specific analysis results are shown in (Table 2).

Table 2. Reliability and Validity Test

\begin{tabular}{|l|l|l|l|l|l|l|}
\hline Variable & Items & $\begin{array}{l}\text { Factor } \\
\text { Loading }\end{array}$ & Eigenvalues & $\begin{array}{l}\text { Percentage } \\
\text { of } \\
\text { variance } \\
\text { explained }\end{array}$ & KMO & $\begin{array}{l}\text { Cronbach's } \\
\text { Alpha }\end{array}$ \\
\hline \multirow{5}{*}{$\begin{array}{l}\text { Job } \\
\text { Satisfaction }\end{array}$} & JS1 & .563 & 5.760 & 30.318 & & \\
\cline { 2 - 5 } & JS2 & .739 & 1.387 & 7.302 & & \\
\cline { 2 - 5 } & JS3 & .554 & 1.262 & 6.641 & \\
\cline { 2 - 5 } & JS4 & .490 & 1.168 & 6.147 & \\
\cline { 2 - 5 } & JS5 & .539 & 1.152 & 6.066 & \\
\cline { 2 - 5 } & JS6 & .628 & 1.006 & 5.297 & \multirow{3}{*}{$\mathbf{0 . 8 4 5}$} \\
\cline { 2 - 5 } & JS & .564 & .932 & 4.907 & \\
\hline
\end{tabular}




\begin{tabular}{|c|c|c|c|c|c|c|}
\hline & JS10 & .394 & .699 & 3.677 & & \\
\hline & JS11 & .535 & .658 & 3.462 & & \\
\hline & JS12 & .514 & .555 & 2.922 & & \\
\hline & JS13 & .546 & .535 & 2.814 & & \\
\hline & JS14 & .455 & .505 & 2.656 & & \\
\hline & JS15 & .589 & .428 & 2.253 & & \\
\hline & JS16 & .554 & .419 & 2.207 & & \\
\hline & JS17 & .537 & .390 & 2.054 & & \\
\hline & JS18 & .551 & .337 & 1.772 & & \\
\hline & JS19 & .558 & .283 & 1.491 & & \\
\hline \multirow{18}{*}{$\begin{array}{l}\text { Employee } \\
\text { Turnover }\end{array}$} & ET1 & .556 & 4.278 & 23.769 & \multirow{18}{*}{0.692} & \multirow{18}{*}{.801} \\
\hline & ET2 & .549 & 1.730 & 9.612 & & \\
\hline & ET3 & .319 & 1.476 & 8.198 & & \\
\hline & ET4 & .347 & 1.365 & 7.581 & & \\
\hline & ET5 & .490 & 1.275 & 7.085 & & \\
\hline & ET6 & .523 & 1.060 & 5.889 & & \\
\hline & ET7 & .526 & .970 & 5.390 & & \\
\hline & ET8 & .424 & .910 & 5.056 & & \\
\hline & ET9 & .627 & .762 & 4.234 & & \\
\hline & ET10 & .536 & .713 & 3.963 & & \\
\hline & ET11 & .458 & .608 & 3.380 & & \\
\hline & ET12 & .541 & .559 & 3.108 & & \\
\hline & ET13 & .429 & .497 & 2.763 & & \\
\hline & ET14 & .497 & .474 & 2.634 & & \\
\hline & ET15 & .568 & .398 & 2.211 & & \\
\hline & ET16 & .616 & .369 & 2.048 & & \\
\hline & ET17 & .346 & .311 & 1.730 & & \\
\hline & ET18 & .198 & .243 & 1.351 & & \\
\hline
\end{tabular}

Source: Estimated

\section{Correlation Analysis}

The correlation was analyzed to verify the relationship between the variables before the hypothesis test. If the correlation coefficient's absolute value is between 0.2 and 0.4 , then the correlation is less. If the correlation coefficients are 0.4 and 0.6 , then the correlation is more significant. The correlation is strong if the correlation coefficient is 0.6 or more. (Table 3 ) shows that the correlation coefficient between each variable is greater than 0.2 , which is significant at the level of $p<0.05$. In previous studies, it was generally considered that there was a possibility of multiple intersections when the correlation was 0.8 or higher. In this study, the maximum correlation coefficient does not exceed 0.7 , so there are no problems that can cause multiple linings between variables. 
Table 3. Correlation Analysis

\begin{tabular}{|c|c|c|c|c|c|c|c|c|c|c|c|}
\hline \multicolumn{12}{|c|}{ Correlations } \\
\hline & & JS-1 & JS-2 & JS-3 & JS-4 & JS-5 & ET-1 & ET-2 & ET-3 & ET-4 & ET-5 \\
\hline \multirow[t]{6}{*}{$\begin{array}{l}\text { JS- } \\
1\end{array}$} & \begin{tabular}{|l|} 
Pearson \\
Correlati \\
on \\
\end{tabular} & 1 & .538 & .359 & .250 & .376 & .487 & .390 & .218 & .189 & .221 \\
\hline & $\begin{array}{l}\text { Sig. (1- } \\
\text { tailed) }\end{array}$ & & .000 & .000 & .001 & .000 & .000 & .000 & .004 & .011 & .003 \\
\hline & Sum of & 62.75 & 31.89 & 20.08 & 15.67 & 26.00 & 35.93 & 21.68 & 13.63 & 12.77 & 14.00 \\
\hline & $\begin{array}{l}\text { Squares } \\
\text { and } \\
\text { Cross- } \\
\text { products }\end{array}$ & 2 & 9 & 1 & 1 & 0 & 3 & 5 & 8 & 2 & 0 \\
\hline & $\begin{array}{l}\text { Covarian } \\
\text { ce }\end{array}$ & .424 & .216 & .136 & .106 & .176 & .243 & .147 & .092 & .086 & .095 \\
\hline & $\mathrm{N}$ & 149 & 149 & 149 & 149 & 149 & 149 & 149 & 149 & 149 & 149 \\
\hline \multirow[t]{6}{*}{$\begin{array}{l}\text { JS- } \\
2\end{array}$} & \begin{tabular}{|l|} 
Pearson \\
Correlati \\
on \\
\end{tabular} & .538 & 1 & .344 & .215 & .260 & .431 & .379 & .199 & -.005 & .017 \\
\hline & $\begin{array}{l}\text { Sig. (1- } \\
\text { tailed) }\end{array}$ & .000 & & .000 & .004 & .001 & .000 & .000 & .008 & .477 & .420 \\
\hline & Sum of & 31.89 & 56.04 & 18.16 & 12.73 & 17.00 & 30.02 & 19.92 & 11.74 & -.309 & 1.000 \\
\hline & $\begin{array}{l}\text { Squares } \\
\text { and } \\
\text { Cross- } \\
\text { products }\end{array}$ & 9 & 0 & 8 & 2 & 0 & 7 & 6 & 5 & & \\
\hline & $\begin{array}{l}\text { Covarian } \\
\text { ce } \\
\end{array}$ & .216 & .379 & .123 & .086 & .115 & .203 & .135 & .079 & -.002 & .007 \\
\hline & $\mathrm{N}$ & 149 & 149 & 149 & 149 & 149 & 149 & 149 & 149 & 149 & 149 \\
\hline \multirow[t]{5}{*}{$\begin{array}{l}\text { JS- } \\
3\end{array}$} & \begin{tabular}{|l|} 
Pearson \\
Correlati \\
on \\
\end{tabular} & .359 & .344 & 1 & .434 & .211 & .346 & .159 & .172 & .150 & .177 \\
\hline & $\begin{array}{l}\text { Sig. (1- } \\
\text { tailed) }\end{array}$ & .000 & .000 & & .000 & .005 & .000 & .027 & .018 & .034 & .015 \\
\hline & $\begin{array}{l}\text { Sum of } \\
\text { Squares } \\
\text { and } \\
\text { Cross- } \\
\text { products }\end{array}$ & $\begin{array}{r}20.08 \\
1\end{array}$ & $\begin{array}{r}18.16 \\
8\end{array}$ & $\begin{array}{r}49.86 \\
6\end{array}$ & $\begin{array}{r}24.21 \\
5\end{array}$ & $\begin{array}{r}13.00 \\
0\end{array}$ & $\begin{array}{r}22.77 \\
9\end{array}$ & 7.859 & 9.604 & 9.047 & $\begin{array}{r}10.00 \\
0\end{array}$ \\
\hline & $\begin{array}{l}\text { Covarian } \\
\text { ce }\end{array}$ & .136 & .123 & .337 & .164 & .088 & .154 & .053 & .065 & .061 & .068 \\
\hline & $\mathrm{N}$ & 149 & 149 & 149 & 149 & 149 & 149 & 149 & 149 & 149 & 149 \\
\hline $\begin{array}{l}\text { JS- } \\
4\end{array}$ & \begin{tabular}{|l|} 
Pearson \\
Correlati \\
on \\
\end{tabular} & .250 & .215 & .434 & 1 & .348 & .152 & .177 & .193 & .114 & .063 \\
\hline
\end{tabular}




\begin{tabular}{|c|c|c|c|c|c|c|c|c|c|c|c|}
\hline & $\begin{array}{l}\text { Sig. (1- } \\
\text { tailed) }\end{array}$ & .001 & .004 & .000 & & .000 & .032 & .015 & .009 & .082 & .222 \\
\hline & Sum of & 15.67 & 12.73 & 24.21 & 62.45 & 24.00 & 11.15 & 9.826 & 12.03 & 7.725 & 4.000 \\
\hline & $\begin{array}{l}\text { Squares } \\
\text { and } \\
\text { Cross- } \\
\text { products }\end{array}$ & 1 & 2 & 5 & 6 & 0 & 4 & & 4 & & \\
\hline & $\begin{array}{l}\text { Covarian } \\
\text { ce }\end{array}$ & .106 & .086 & .164 & .422 & .162 & .075 & .066 & .081 & .052 & .027 \\
\hline & $\mathrm{N}$ & 149 & 149 & 149 & 149 & 149 & 149 & 149 & 149 & 149 & 149 \\
\hline $\begin{array}{l}\text { JS- } \\
5\end{array}$ & $\begin{array}{l}\text { Pearson } \\
\text { Correlati } \\
\text { on }\end{array}$ & .376 & .260 & .211 & .348 & 1 & .246 & .327 & .218 & .148 & .201 \\
\hline & $\begin{array}{l}\text { Sig. (1- } \\
\text { tailed) }\end{array}$ & .000 & .001 & .005 & .000 & & .001 & .000 & .004 & .036 & .007 \\
\hline & Sum of & 26.00 & 17.00 & 13.00 & 24.00 & 76.00 & 20.00 & 20.00 & 15.00 & 11.00 & 14.00 \\
\hline & $\begin{array}{l}\text { Squares } \\
\text { and } \\
\text { Cross- } \\
\text { products }\end{array}$ & 0 & 0 & 0 & 0 & 0 & 0 & 0 & 0 & 0 & 0 \\
\hline & $\begin{array}{l}\text { Covarian } \\
\text { ce }\end{array}$ & .176 & .115 & .088 & .162 & .514 & .135 & .135 & .101 & .074 & .095 \\
\hline & $\mathrm{N}$ & 149 & 149 & 149 & 149 & 149 & 149 & 149 & 149 & 149 & 149 \\
\hline $\begin{array}{l}\text { ET } \\
-1\end{array}$ & $\begin{array}{l}\text { Pearson } \\
\text { Correlati } \\
\text { on }\end{array}$ & .487 & .431 & .346 & .152 & .246 & 1 & .392 & .088 & .165 & .228 \\
\hline & $\begin{array}{l}\text { Sig. (1- } \\
\text { tailed) }\end{array}$ & .000 & .000 & .000 & .032 & .001 & & .000 & .142 & .022 & .003 \\
\hline & Sum of & 35.93 & 30.02 & 22.77 & 11.15 & 20.00 & 86.68 & 25.61 & 6.497 & 13.12 & 17.00 \\
\hline & $\begin{array}{l}\text { Squares } \\
\text { and } \\
\text { Cross- } \\
\text { products }\end{array}$ & 3 & 7 & 9 & 4 & 0 & 5 & 7 & & 8 & 0 \\
\hline & $\begin{array}{l}\text { Covarian } \\
\text { ce }\end{array}$ & .243 & .203 & .154 & .075 & .135 & .586 & .173 & .044 & .089 & .115 \\
\hline & $\mathrm{N}$ & 149 & 149 & 149 & 149 & 149 & 149 & 149 & 149 & 149 & 149 \\
\hline $\begin{array}{l}\text { ET } \\
-2\end{array}$ & $\begin{array}{l}\text { Pearson } \\
\text { Correlati } \\
\text { on } \\
\end{array}$ & .390 & .379 & .159 & .177 & .327 & .392 & 1 & .255 & -.085 & .125 \\
\hline & $\begin{array}{l}\text { Sig. (1- } \\
\text { tailed) }\end{array}$ & .000 & .000 & .027 & .015 & .000 & .000 & & .001 & .151 & .065 \\
\hline & $\begin{array}{l}\text { Sum of } \\
\text { Squares } \\
\text { and } \\
\text { Cross- } \\
\text { products }\end{array}$ & $\begin{array}{r}21.68 \\
5\end{array}$ & $\begin{array}{r}19.92 \\
6\end{array}$ & 7.859 & 9.826 & $\begin{array}{r}20.00 \\
0\end{array}$ & $\begin{array}{r}25.61 \\
7\end{array}$ & $\begin{array}{r}49.30 \\
2\end{array}$ & $\begin{array}{r}14.13 \\
4\end{array}$ & 5.101 & 7.000 \\
\hline
\end{tabular}




\begin{tabular}{|c|c|c|c|c|c|c|c|c|c|c|c|}
\hline & $\begin{array}{l}\text { Covarian } \\
\text { ce }\end{array}$ & .147 & .135 & .053 & .066 & .135 & .173 & .333 & .096 & -.034 & .047 \\
\hline & $\mathrm{N}$ & 149 & 149 & 149 & 149 & 149 & 149 & 149 & 149 & 149 & 149 \\
\hline \multirow[t]{5}{*}{$\begin{array}{l}\text { ET } \\
-3\end{array}$} & $\begin{array}{l}\text { Pearson } \\
\text { Correlati } \\
\text { on }\end{array}$ & .218 & 199 & .172 & .193 & .218 & .088 & .255 & 1 & .212 & -.143 \\
\hline & $\begin{array}{l}\text { Sig. (1- } \\
\text { tailed) }\end{array}$ & .004 & .008 & .018 & .009 & .004 & .142 & .001 & & .005 & .041 \\
\hline & $\begin{array}{l}\text { Sum of } \\
\text { Squares } \\
\text { and } \\
\text { Cross- } \\
\text { products }\end{array}$ & $\begin{array}{r}13.63 \\
8\end{array}$ & $\begin{array}{r}11.74 \\
5\end{array}$ & 9.604 & $\begin{array}{r}12.03 \\
4\end{array}$ & $\begin{array}{r}15.00 \\
0\end{array}$ & 6.497 & $\begin{array}{r}14.13 \\
4\end{array}$ & $\begin{array}{r}62.28 \\
2\end{array}$ & $\begin{array}{r}14.28 \\
9\end{array}$ & 9.000 \\
\hline & $\begin{array}{l}\text { Covarian } \\
\text { ce }\end{array}$ & .092 & .079 & .065 & .081 & .101 & .044 & .096 & .421 & .097 & -.061 \\
\hline & $\mathrm{N}$ & 149 & 149 & 149 & 149 & 149 & 149 & 149 & 149 & 149 & 149 \\
\hline \multirow[t]{5}{*}{$\begin{array}{l}\text { ET } \\
-4\end{array}$} & $\begin{array}{l}\text { Pearson } \\
\text { Correlati } \\
\text { on }\end{array}$ & .189 & -.005 & .150 & .114 & .148 & .165 & -.085 & .212 & 1 & .380 \\
\hline & $\begin{array}{l}\text { Sig. (1- } \\
\text { tailed) }\end{array}$ & .011 & .477 & .034 & .082 & .036 & .022 & .151 & .005 & & .000 \\
\hline & $\begin{array}{l}\text { Sum of } \\
\text { Squares } \\
\text { and } \\
\text { Cross- } \\
\text { products }\end{array}$ & $\begin{array}{r}12.77 \\
2\end{array}$ & -.309 & 9.047 & 7.725 & $\begin{array}{r}11.00 \\
0\end{array}$ & $\begin{array}{r}13.12 \\
8\end{array}$ & 5.101 & $\begin{array}{r}14.28 \\
9\end{array}$ & $\begin{array}{r}73.03 \\
4\end{array}$ & $\begin{array}{r}26.00 \\
0\end{array}$ \\
\hline & $\begin{array}{l}\text { Covarian } \\
\text { ce }\end{array}$ & .086 & -.002 & .061 & .052 & .074 & .089 & -.034 & .097 & .493 & .176 \\
\hline & $\mathrm{N}$ & 149 & 149 & 149 & 149 & 149 & 149 & 149 & 149 & 149 & 149 \\
\hline \multirow[t]{5}{*}{$\begin{array}{l}\text { ET } \\
-5\end{array}$} & $\begin{array}{l}\text { Pearson } \\
\text { Correlati } \\
\text { on } \\
\end{array}$ & .221 & .017 & .177 & .063 & .201 & .228 & .125 & -.143 & .380 & 1 \\
\hline & $\begin{array}{l}\text { Sig. (1- } \\
\text { tailed) }\end{array}$ & .003 & .420 & .015 & .222 & .007 & .003 & .065 & .041 & .000 & \\
\hline & $\begin{array}{l}\text { Sum of } \\
\text { Squares } \\
\text { and } \\
\text { Cross- } \\
\text { products }\end{array}$ & $\begin{array}{r}14.00 \\
0\end{array}$ & 1.000 & $\begin{array}{r}10.00 \\
0\end{array}$ & 4.000 & $\begin{array}{r}14.00 \\
0\end{array}$ & $\begin{array}{r}17.00 \\
0\end{array}$ & 7.000 & $\begin{array}{r}- \\
9.000\end{array}$ & $\begin{array}{r}26.00 \\
0\end{array}$ & $\begin{array}{r}64.00 \\
0\end{array}$ \\
\hline & $\begin{array}{l}\text { Covarian } \\
\text { ce }\end{array}$ & .095 & .007 & .068 & .027 & .095 & .115 & .047 & -.061 & .176 & .432 \\
\hline & $\mathrm{N}$ & 149 & 149 & 149 & 149 & 149 & 149 & 149 & 149 & 149 & 149 \\
\hline
\end{tabular}




\section{Hypothesis Validation}

This study uses SPSS and regression analysis to identify the relationship between the Dromotropic factor and job satisfaction. If the R-squared is 0.011 , it indicates that the input variables explain $1.1 \%$ of the input variables. But this value is meager R-squared means this model, not a better fit for this analysis. The Dromotropic factor did not impact any job satisfaction and employee turnover. So, This Hypothesis was valid.

Table 4. Hypothesis Validation

\begin{tabular}{|c|c|c|c|c|c|c|c|c|}
\hline \multirow{2}{*}{$\begin{array}{c}\mathrm{R} \\
\text { Square }\end{array}$} & \multirow{2}{*}{$\begin{array}{l}\text { Adjusted } \\
\text { R Square }\end{array}$} & \multirow{2}{*}{$\begin{array}{l}\text { Std. Error } \\
\text { of the } \\
\text { Estimate }\end{array}$} & \multicolumn{5}{|c|}{ Change Statistics } & \multirow[t]{2}{*}{ Durbin-Watson } \\
\hline & & & $\begin{array}{c}\text { R } \\
\text { Square } \\
\text { Change }\end{array}$ & $\begin{array}{c}\mathrm{F} \\
\text { Change }\end{array}$ & df1 & df2 & $\begin{array}{c}\text { Sig. F } \\
\text { Change }\end{array}$ & \\
\hline .011 & -.016 & 1.190 & .011 & .406 & 4 & 144 & .804 & .044 \\
\hline
\end{tabular}

\begin{tabular}{|c|c|c|c|c|c|c|c|c|c|c|}
\hline \multirow[b]{2}{*}{ Model } & \multicolumn{2}{|c|}{$\begin{array}{l}\text { Unstandardized } \\
\text { Coefficients }\end{array}$} & \multirow{2}{*}{\begin{tabular}{|c|}
$\begin{array}{c}\text { Standardized } \\
\text { Coefficients }\end{array}$ \\
Beta \\
\end{tabular}} & \multirow[b]{2}{*}{$\mathrm{t}$} & \multirow[b]{2}{*}{ Sig. } & \multicolumn{3}{|c|}{ Correlations } & \multicolumn{2}{|c|}{$\begin{array}{c}\text { Collinearity } \\
\text { Statistics }\end{array}$} \\
\hline & B & $\begin{array}{l}\text { Std. } \\
\text { Error }\end{array}$ & & & & $\begin{array}{l}\text { Zero- } \\
\text { order }\end{array}$ & Partial & Part & Tolerance & VIF \\
\hline 1 (Constant) & 2.914 & .890 & & 3.275 & .001 & & & & & \\
\hline $\begin{array}{l}\text { Are you satisfied } \\
\text { with the } \\
\text { compensation } \\
\text { and benefits } \\
\text { offered to you by } \\
\text { your current } \\
\text { employer? }\end{array}$ & -.144 & .143 & -.089 & 1.005 & .317 & -.071 & -.083 & .083 & .883 & 1.132 \\
\hline $\begin{array}{l}\text { Were you } \\
\text { satisfied with the } \\
\text { employee }\end{array}$ & & & & & & & & & & \\
\hline $\begin{array}{l}\text { retention } \\
\text { measures } \\
\text { implemented in } \\
\text { your } \\
\text { Organization? }\end{array}$ & .105 & .148 & .063 & .708 & .480 & .029 & .059 & .059 & .855 & 1.170 \\
\hline $\begin{array}{l}\text { How do you rate } \\
\text { your on-the-job } \\
\text { training and }\end{array}$ & & & & & & & & & & \\
\hline $\begin{array}{l}\text { Induction } \\
\text { experience in the } \\
\text { current } \\
\text { organization? }\end{array}$ & .053 & .170 & .027 & .311 & .756 & .007 & .026 & .026 & .878 & 1.139 \\
\hline
\end{tabular}




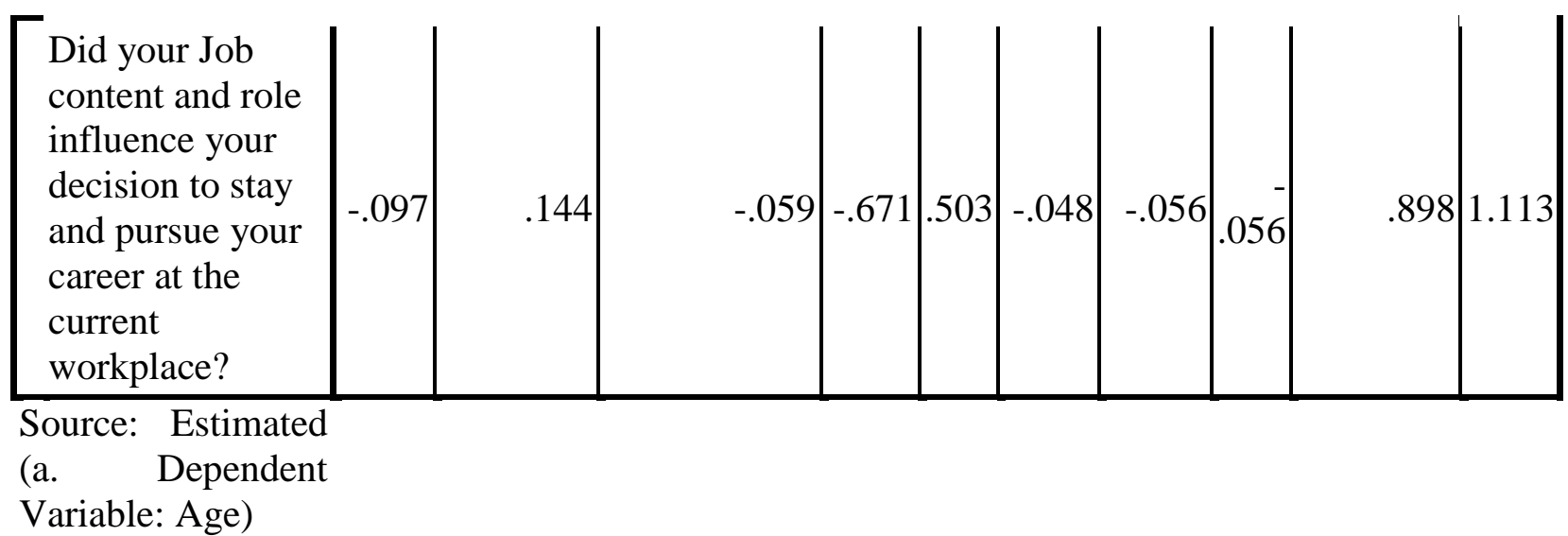

\section{CONCLUSION \& POLICY IMPLICATIONS}

The analysis aims to define the link between employee happiness and turnover. We found that the test findings were broadly in line with the theories and the hypotheses backed by observational research. The analytical analyses are summed up as follows: Firstly, the study results indicate that the partnerships between the organization and the turnover targets are both direct and undirected. Employment hopping and turnover culture have been suggested to moderate the interaction between the variable mediator and the variable based. The study results indicate that the dromotropic effect does not influence worker satisfaction and employee turnover in Bangladesh's public and private sectors. But hoping and employee turnover are directly related to human resources policies and benefits and more employment prospects.

\section{REFERENCES}

Arshad, M. A. (2013). Effects of Performance Appraisal Politics on Job Satisfaction, Turnover Intention and Loyalty to Supervisor, International Review of Management and Business Research, 2(3).

Belete, A.K. (2018). Turnover Intention Influencing Factors of Employees: An Empirical Work Review. Journal of Entrepreneurship \& Organization Management.

Chan, B. Y. F., Yeoh, L. C.L. \& Osman, S.(2010). An exploratory study on Turnover Intention among Private Sector Employees, International Journal of Business and Management. 5(8), 57-64.

Cranny, P.C. Smith \& Stone, E. F. (2016). Job Satisfaction: How People Feel About Their Jobs and how it affects Their Performance. New York: Lexington BooksEdwin A. Locke, Job Satisfaction: Overview.

Huak, M., Pivi, F. G., \& Hassan, Z. (2015). The Impact of Organizational Citizenship Behavior on Employee's job satisfaction, commitment and turnover intention in Dining Restaurants Malaysia. International Journal Accounting, Business, and Management, $1(1), 1-17$.

Krishnan, L. R. K. (2012). A Study of Job Hopping and Employee Turnover in the Telecom Service Industry in Tamil Nadu. IJRCM, 2, 9-79. 
Kirschenbaum, A. \& Rita, M.N. (1999). Underlying Labor Market Dimensions of Opportunities: The Case of Employee Turnover. Human Relations (HR), 52(10), 1233 1255.

Lawrence, D. S. (2003). An Unconventional Guide to Surviving Corporate Employment. Palmyra, VA, USA: Bent Crow Press. 13.

Lee, T. R. \& Mitchell, T. W. (2001). The Unfolding Model of Voluntary Turnover and Job Embeddedness: Foundations for a Comprehensive Theory of Attachment. Research in Organizational Behavior, 23, 189-246.

Leedy, J. E. \& Ormrod, P. D. (2001). Practical Research: Planning and Design. Upper Saddle River, New Jersey, USA: Merrill Prentice Hall. Organizations. New York: Wiley.

\section{Copyrights}

Copyright for this article is retained by the author(s), with first publication rights granted to the journal. This is an open-access article distributed under the terms and conditions of the Creative Commons Attribution license (http://creativecommons.org/licenses/by/4.0/) 